\title{
Meeting or Exceeding Physical Activity Guidelines is Associated with Reduced Risk for Cancer in Mexican- Americans
}

\author{
Shenghui Wu' ${ }^{1}$, Susan P. Fisher-Hoch ${ }^{2}$, Belinda Reninger ${ }^{3}$, Joseph B. McCormick ${ }^{2, *}$ \\ ${ }^{1}$ Department of Epidemiology \& Biostatistics, University of Texas Health Science Center at San Antonio-Laredo Campus, \\ Laredo, TX (SW) \\ ${ }^{2}$ Division of Epidemiology, University of Texas Health Science Center-Houston, School of Public Health, Brownsville Campus, \\ Brownsville, TX (SPF, JBM) \\ ${ }^{3}$ Division of Health Promotion and Health Behavior University of Texas Health Science Center-Houston, School of Public Health , \\ Brownsville Campus, Brownsville, TX \\ *Corresponding author: Joseph.b.mccormick@uth.tmc.edu
}

\begin{abstract}
Background: Epidemiologic studies have shown that inadequate physical activity was associated with cancers in whites and other ethnic groups, but in Mexican-Americans data are limited. This study aimed to measure the association between physical activity and reported cancer risk in Mexican-Americans. Methods: Participants were drawn from the Cameron County Hispanic Cohort $(n=3,391)$, a randomly selected Mexican-American cohort in Texas on the US-Mexico border. Physical activity was assessed using the International Physical Activity Questionnaire. Cancer was self-reported by the participants as being told by a health care provider that they had cancer. Results: Ninety-nine participants of the cohort (2.94\%) reported a diagnosis of cancer. Compared to participants who did not meet US physical activity guidelines, subjects who met physical activity guidelines of 150 moderate and vigorous minutes per week ( $\geq 600$ METs) reduced their risk for cancer by $87 \%$ (OR $=0.13$; 95\% CI: $0.03-0.54$ ), and subjects with total minutes per week of moderate and vigorous/strenuous activity greater than 745 METs decreased cancer risk by $86 \%$ [odds ratio $(\mathrm{OR})=0.14$; $95 \%$ confidence interval $(\mathrm{CI}): 0.03-0.60$ ] comparing with their counterparts, after adjusting for age, gender, body mass index, smoking and alcohol drinking status, education and total portions of fruit and vegetable intake. Conclusions: Meeting or exceeding recommended levels of moderate and vigorous physical activity was associated with a significantly reduced risk of reporting cancer by Mexican-Americans. Meeting or exceeding recommended levels of physical activity appears to be an effective target for cancer prevention and control among Mexican-Americans independent of BMI and other factors.
\end{abstract}

Keywords: physical activity, cancer, minority population, Latino, Mexican American

Cite This Article: Shenghui Wu, Susan P. Fisher-Hoch, Belinda Reninger, and Joseph B. McCormick, "Meeting or Exceeding Physical Activity Guidelines is Associated with Reduced Risk for Cancer in MexicanAmericans." American Journal of Cancer Prevention, vol. 4, no. 1 (2016): 1-7. doi: 10.12691/ajcp-4-1-1.

\section{Introduction}

According to the US Census Bureau, 50.5 million Americans, or $16 \%$ of the total US population, identified themselves as Hispanic or Latino in 2010 [1,2]. Cancer is the leading cause of death among Hispanics, accounting for 21\% of deaths [3]. About 1 in 2 Hispanic men and 1 in 3 Hispanic women will be diagnosed with cancer in their lifetime. The lifetime probability of dying from cancer is 1 in 5 for Hispanic men and 1 in 6 for Hispanic women [3]. According to the 2012-2014 statistics from American Cancer Society [3], for all cancers combined, prostate, female breast, colorectal, and lung cancer incidence and death rates are lower among Hispanics than among nonHispanic whites. Cancer rates for stomach, cervix, liver, acute lymphocytic leukemia, and gallbladder are higher in Hispanics. The roles of physical inactivity and obesity in cancer incidence are marked, and a large number of cancer cases and deaths could well be prevented with the adoption of healthier lifestyles, including not smoking, maintaining a healthy body weight, and being physically active [3]. Epidemiologic studies have shown that inadequate physical activity was associated with many cancers in whites and other ethnic groups, but in MexicanAmericans data are limited and not all studies reported statistically significant associations [4]. One case-control study [5] found a significant protective effect of physical activity on breast cancer risk among Hispanic women. High levels of total non-occupational activity around the time of diagnosis reduced $71 \%$ of breast cancer risk among Hispanic premenopausal women, but not among non-Hispanic whites [5]. In contrast, another case-control study reported a non-significant protective effect of physical activity on breast cancer risk in Hispanic women [6]. Another case-control study showed that physical activity decreased the risk for endometrial cancer by 53\% 
compared to physical inactivity among Mexican women in Mexico City [7], but no epidemiological studies were available among American Hispanic populations. The objective of this study was to explore the effect of physical activity on general cancer risk in a randomly selected cohort of Mexican-American subjects.

\section{Materials and Methods}

\subsection{Study Participants}

This study was approved by the Committee for the Protection of Human Subjects of the UT Health, Houston and the Institutional Review Board of the University of the Texas Health Science Center, San Antonio. All study participants gave written informed consent. This crosssectional analysis used data from the Cameron County Hispanic Cohort (CCHC), an ongoing homogenous community-dwelling Mexican-American cohort study $[8,9]$. Study subjects were recruited from randomly selected tract/blocks according to the 2000 Census as described previously [8,9]. At the baseline survey conducted between 2003 and 2015, 3,391 participants aged 18 years or older were recruited from their households in predominantly Mexican-American cities along the Rio Grande border with Mexico (all participants recruited from Brownsville from 2003 to 2012).

All subjects responded to a detailed baseline survey of demographic characteristics, lifestyle including diet, physical activity, family and medical history, and other exposures. Participants were asked to fast for at least 10 hours overnight before a clinic visit at the Clinical Research Unit. Anthropometric measurements, including current weight, height, and circumferences of the waist and hip, were also taken [8,9].

Physical activity in a typical week according to intensity, frequency (times / week) and duration (minutes / time) was assessed using the International Physical Activity Questionnaire short-form (IPAQ) [10] or the Godin Leisure-Time Exercise Questionnaire instruments [11] as reported previously [12]. The instruments have reported evidence of reliability and validity $[10,13,14,15,16]$. Metabolic equivalent adjusted minutes (MET adjusted minutes) of moderate and vigorous physical activity in the last week was calculated based on responses [11]. The MET intensity of physical activity was classified as light intensity (< 3 METs), moderate intensity (3-6 METs), and vigorous intensity ( $>6$ METs) [11]. Physical activity $\geq 600$ MET adjusted minutes was considered meeting United States physical activity guidelines (USDHHS, 2008) [17].

All participants responded to a detailed baseline survey that collected information on demographic characteristics, lifestyle and dietary histories, medical history, and other exposures. Using the Food Frequency Questionnaire[18], fruit and vegetable consumption was assessed by asking participants how many portions of fruit and vegetables they ate daily. A portion size was described as a $1 / 2$ cup of fresh, frozen, or canned produce or a medium-sized piece of produce (such as melon with 7-8 inches in diameter) $[19,20]$. Consumption of five or more fruit and vegetable portions was considered meeting US guidelines (U.S. Department of Health and Human Services)[19,20]. Weight was measured to the nearest tenth of a kilogram and height to the nearest tenth of a centimeter. Body mass index (BMI) was calculated as weight in kilograms divided by height squared in meters $\left(\mathrm{kg} / \mathrm{m}^{2}\right)$. Waist circumference (WC) was measured at the level of the umbilicus and hip circumference (HC) at the level of maximum width of the buttocks with participants in a standing position and breathing normally, to the nearest $0.2 \mathrm{~cm}$. Waist-to-hip ratio (WHR) was calculated as WC divided by HC [8]. Body fat percentage was estimated using the resistance values from the Quantum bioelectric body composition analyzer with the sex-specific equations from Sun et al [21]. The average of 3 blood pressures (BP) taken 5 minutes apart were used.

\subsection{Laboratory Measurements}

All participants provided a blood sample at baseline. After collection, samples were placed on ice and centrifuged within 30 minutes. Following processing and aliquoting, all samples were stored at $-80^{\circ} \mathrm{C}$ until laboratory analyses were conducted. Laboratory studies performed included fasting lipid panel, hemoglobin $(\mathrm{Hb})$ A1c and fasting plasma glucose all performed by a local CLIA certified laboratory. Fasting serum insulin was consistently performed in-house using Mercodia immunoassays (Uppsala, Sweden). Homeostasis model assessment insulin resistance index (HOMA-IR) was calculated as fasting glucose $(\mathrm{mg} / \mathrm{dL}) / 18 \times$ fasting insulin $(\mathrm{mU} / \mathrm{L}) / 22.5$ [22]. High sensitivity C-reactive protein (hsCRP) levels were measured using Quantikine ${ }^{\circledR}$ ELISA kit (R \& D Systems, Inc., Minneapolis, USA).

\subsection{Identification of Cancer}

Cancer diagnosis was identified in participants as being told by a health care provider that they had cancer.

\subsection{Statistical Analysis}

Descriptive results and the models reported in this paper were adjusted for the probability sampling weights taking into consideration clustering effects arising from the census block and household [8]. Log-transformation was conducted to normalize the distribution of biomarkers as appropriate. Survey-weighted linear regression was used to obtain the t-test statistics to compare phenotypes and to be used for multiple pairwise mean comparisons for continuous data. Survey-weighted chi-square test was used to obtain Rao-Scott F adjusted chi-square statistic to compare phenotypes for categorical data. Survey-weighted logistic regression modeling was performed to estimate the ORs for cancer risk and their 95\% CIs by physical activity adjusting for other covariates. Potential confounders adjusted for in multivariable survey-weighted logistic regression models included age, gender, education, obesity, alcohol drinking and cigarette smoking status, servings of fruits and vegetables per day and discrepancies in survey versions for physical activity data collection. Physical activity was classified into two groups respectively if subjects met physical activity guidelines of 150 moderate and vigorous minutes per week $(\geq 600$ METs), and according to the median of the total minutes per week of moderate and vigorous/strenuous activity (745 METs). 
Statistical analyses were carried out by using SAS version 9.3 (SAS Institute, Cary, NC). All statistical tests were based on 2-sided probability.

\section{Results}

At the time of this study a total of 3,391 individuals were enrolled in the CCHC, 2,930 from Brownsville and 266 participants from Harlingen (Lower Rio Grande
Valley) and 195 participants from Laredo (Webb County), Texas. Based on the recommended scoring protocols, 29 $(0.9 \%)$ participants with extreme values $(\geq 7,680$ MET adjusted minutes) of physical activity were excluded from the analyses. Of remaining 3,362 participants, mean age of this subset was 45 years; $35 \%$ were male. A total of $12.5 \%$ $(n=421)$ of the participants met minimum recommendations for physical activity of $\geq 600$ MET-minutes/week.

Table 1. Cohort Demographics and Metabolic Characteristics Stratified by Cancer Risk: Cameron County Hispanic Cohort Study (20032015) $)^{\mathrm{a}, \mathrm{b}}$

\begin{tabular}{|c|c|c|c|}
\hline \multirow[b]{2}{*}{ Variable } & \multicolumn{3}{|c|}{ Cancer } \\
\hline & $\begin{array}{l}\text { Yes } \\
(\mathrm{n}=99,2.94 \%)\end{array}$ & $\begin{array}{l}\text { No } \\
(n=3,234,96.19 \%)\end{array}$ & $P$-value \\
\hline \multicolumn{4}{|l|}{ Categorical variables, $\mathrm{n}(\%)$} \\
\hline Men & $32(32.32)$ & $1104(34.14)$ & 0.01 \\
\hline Employed & $34(34.34)$ & $1596(49.35)$ & 0.46 \\
\hline Education, below high school & $50(50.51)$ & $1663(51.42)$ & 0.23 \\
\hline Met minimum recommendations for physical activity of $\geq 600$ MET-minutes/week & $7(7.01)$ & $406(12.55)$ & $<.0001$ \\
\hline Met recommendations of $\geq 5$ servings of fruit $\&$ vegetables per day & $3(3.03)$ & $143(4.41)$ & $<.0001$ \\
\hline Current smokers & $10(10.10)$ & $489(15.12)$ & 0.01 \\
\hline Ever smokers & $31(32.63)$ & $971(30.37)$ & 0.04 \\
\hline Ever alcohol drinkers & $58(69.88)$ & $1653(57.58)$ & 0.37 \\
\hline Family history of cancer & $20(20.20)$ & $768(23.68)$ & 0.11 \\
\hline \multicolumn{4}{|l|}{ Continuous variables, Mean (SE) } \\
\hline Age at enrollment (years) & $60.84(4.4)$ & $46.91(1.2)$ & 0.003 \\
\hline Annual household income (US dollars) & 17239 (5589.18) & 35570 (4508.8) & 0.01 \\
\hline MET minutes/wk. of all activity & $288.31(84.94)$ & $968.2(127.4)$ & 0.0004 \\
\hline MET minutes/wk. of moderate and vigorous activity & $100.04(54.48)$ & $850.84(80.78)$ & $<.0001$ \\
\hline MET adjusted minutes of mild activity & $108.69(37.62)$ & $292.19(49.31)$ & 0.002 \\
\hline Average hours per day doing heavy activity & $0.46(0.21)$ & $1.07(0.07)$ & 0.004 \\
\hline Body mass index $\left(\mathrm{kg} / \mathrm{m}^{2}\right)$ & $33.46(0.88)$ & $31.48(0.46)$ & 0.03 \\
\hline Total portions of fruit and vegetables & $2.03(0.24)$ & $2.34(0.14)$ & 0.20 \\
\hline Waist circumference (cm) & $109.84(2.58)$ & $104.90(1.14)$ & 0.08 \\
\hline Waist-to-hip ratio & $0.95(0.02)$ & $0.94(0.01)$ & 0.63 \\
\hline Body fat (\%) & $54.52(9.7)$ & $34.01(0.80)$ & 0.03 \\
\hline Total cholesterol (mg/dL) & $188.06(6.15)$ & $183.78(2.93)$ & 0.43 \\
\hline Triglycerides (mg/dL) & $132.33(21.92)$ & $142.59(4.67)$ & 0.63 \\
\hline HDL-cholesterol (mg/dL) & $55.4(3.12)$ & $48.23(0.74)$ & 0.03 \\
\hline LDL-cholesterol (mg/dL) & $92.35(12.35)$ & $108.58(2.38)$ & 0.20 \\
\hline High sensitivity C-reactive protein $(\mathrm{mg} / \mathrm{L})^{\mathrm{c}}$ & $4.14(1.39)$ & $4.21(1.05)$ & 0.98 \\
\hline Systolic blood pressure (mmHg) & $130.33(4.23)$ & $125.01(1.54)$ & 0.23 \\
\hline Diastolic blood pressure (mmHg) & $78.26(0.96)$ & $76.75(0.52)$ & 0.16 \\
\hline Insulin $(\mathrm{mg} / \mathrm{dL})^{\mathrm{c}}$ & $13.74(1.15)$ & $12.18(1.02)$ & 0.34 \\
\hline Fasting blood glucose $(\mathrm{mg} / \mathrm{dL})^{\mathrm{c}}$ & $101.49(1.04)$ & $105.31(1.02)$ & 0.49 \\
\hline HOMA IR ${ }^{c}$ & $3.86(1.14)$ & $3.16(1.02)$ & 0.13 \\
\hline HbA1c $(\%)^{c}$ & $5.75(1.03)$ & $6.06(1.01)$ & 0.11 \\
\hline
\end{tabular}

${ }^{a}$ Abbreviation: LDL: low-density lipoprotein; Hb: hemoglobin; HDL: high-density lipoprotein; HOMA IR: homeostatic model assessment insulin resistance; MET: metabolic equivalent

${ }^{\mathrm{b}}$ All descriptive results and the models were adjusted for the probability of sampling using weights taking into consideration clustering effects arising from the same census block and household. Linear regression models were used for continuous variables and Rao-Scott F adjusted chi-square statistic for categorical variables.

${ }^{c}$ Geometric concentrations.

Ninety-nine participants of the cohort (2.94\%) reported cancer and 3,234 did not report cancer (Figure 1 and Table 1). Subjects with cancer were more likely to be older, female, cigarette smoking, and less likely to meet the recommended guidelines for physical activity of more than 600 MET-minutes/week or meet the recommended guidelines for fruit and vegetables more than 5 servings per day. They had lower household income but higher BMI and body fat percentage than their counterparts (all $P$ s $<0.05)$. They showed significantly elevated mean values of total cholesterol compared with subjects without cancer, but decreased values of high density lipid cholesterol (HDLC). Although levels of WC, WHR, systolic and diastolic blood pressure, insulin, HOMA-IR and HbA1c were higher in subjects with cancer than those without, the differences were not statistically significant. There was no difference in employment status, education level and alcohol drinking between subjects with and without cancer. Table 2 showed the frequency of specific cancer cites. Seven subjects had breast cancer and 50 subjects had unknown cancer types. Figure 1 provided information about the difference of common influencing 
factors between cancer and non-cancer groups, which suggested that the subjects with cancer had high frequencies or means of common risk factors of cancer but lower ones of protective factors for cancer compared with those without cancer.

Subjects who met physical activity guidelines of 150 moderate and vigorous minutes per week ( $\geq 600$ METs) reduced their risk for cancer by $86 \%(\mathrm{OR}=0.14$; $95 \% \mathrm{CI}$ : 0.04-0.50) after adjusting for age and gender compared with those did not meet guidelines (Table 3). Multivariable-adjusted model showed that the risk was reduced by $87 \% \quad(\mathrm{OR}=0.13 ; 95 \% \quad \mathrm{CI}$ : $0.03-0.57)$. Compared to subjects with total minutes per week of moderate and vigorous/strenuous activity less than 745 METs, subjects with those greater than 745 METs were associated with an $84 \%$ (OR=0.16; 95\% CI: 0.04-0.57) lower risk of cancer after adjusting for age and gender (Table 3). Multivariable-adjusted model showed that the risk was reduced by $86 \%(\mathrm{OR}=0.14$; 95\% CI: 0.03-0.63).
Table 2. Cancer types by specific sites: Cameron County Hispanic Cohort Study (2003-2015)

\begin{tabular}{lll}
\hline Cancer Type & Frequency & Percentage \\
\hline Breast cancer & 7 & $7.07 \%$ \\
Prostate cancer & 6 & $6.06 \%$ \\
Cervical cancer & 5 & $5.05 \%$ \\
Uterus cancer & 4 & $4.04 \%$ \\
Colon cancer & 3 & $3.03 \%$ \\
Uterine cancer & 3 & $10.10 \%$ \\
Melanoma & 3 & $3.03 \%$ \\
Skin cancer & 3 & $3.03 \%$ \\
Brain cancer & 2 & $2.02 \%$ \\
Endometrial cancer & 2 & $2.02 \%$ \\
Leukemia & 2 & $2.02 \%$ \\
Testicular cancer & 2 & $2.02 \%$ \\
Throat cancer & 2 & $2.02 \%$ \\
Esophagus cancer & 1 & $1.01 \%$ \\
Lung cancer & 1 & $1.01 \%$ \\
Kidney cancer & 1 & $1.01 \%$ \\
Spine cancer & 1 & $1.01 \%$ \\
Thyroid cancer & 1 & $1.01 \%$ \\
Unknown cancer & 50 & $50.51 \%$ \\
Total & 99 & $100 \%$ \\
\hline
\end{tabular}

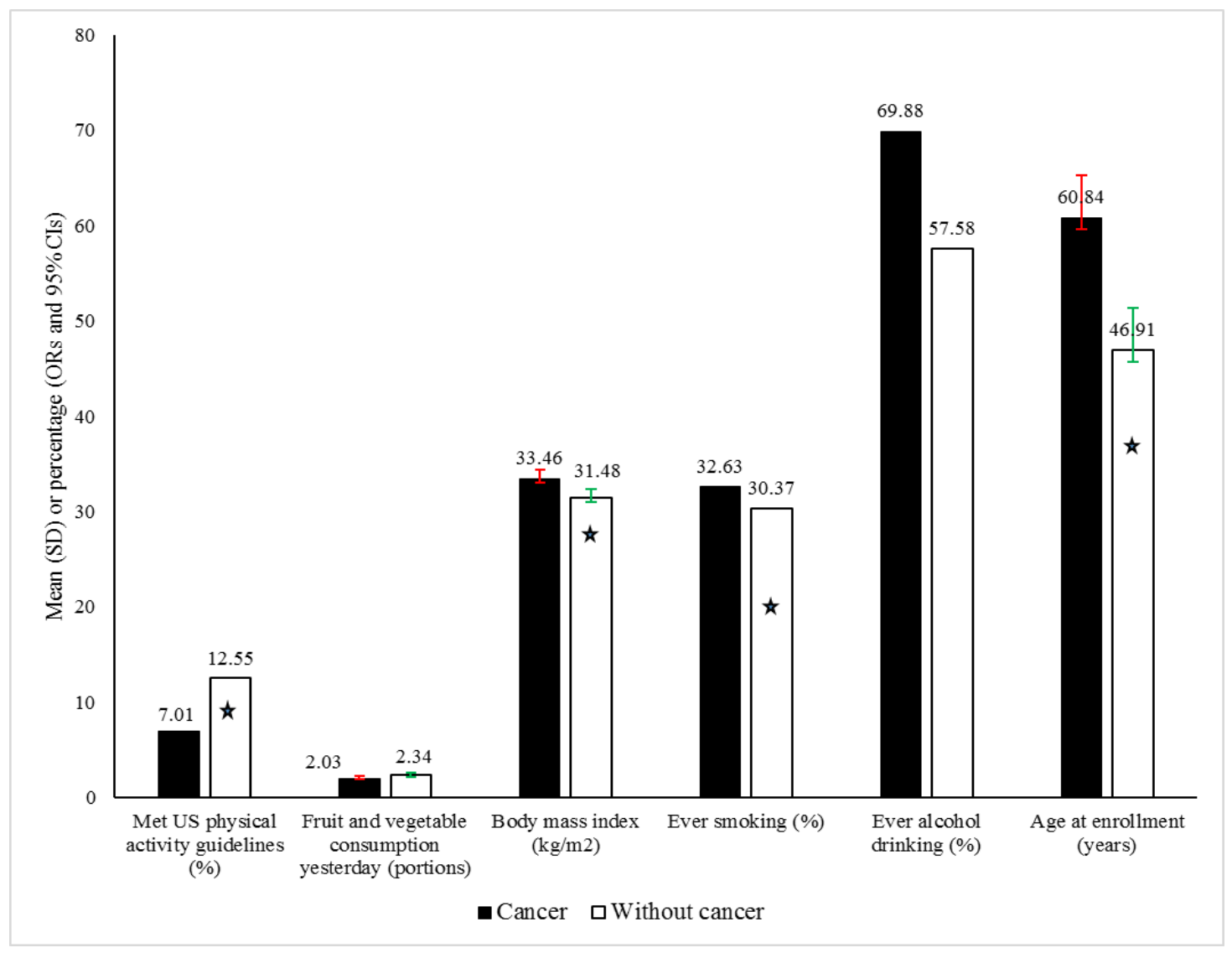

Figure 1. Common influencing factors of cancer in subjects with and without cancer

The star $\star$ indicated that the difference between subjects with and without cancer was statistically significant. SD: standard deviation.

Table 3. Association between physical activity and cancer risk

\begin{tabular}{|c|c|c|c|c|c|c|c|c|c|c|}
\hline \multirow[t]{3}{*}{ Model } & \multicolumn{5}{|c|}{$\begin{array}{c}\text { Meet physical activity guidelines of } 150 \text { moderate and } \\
\text { vigorous minutes per week ( } \geq 600 \text { metabolic equivalents) }\end{array}$} & \multicolumn{5}{|c|}{$\begin{array}{c}\text { Total minutes per week of moderate and } \\
\text { vigorous/strenuous activity ( } \geq 745 \text { metabolic equivalents) }\end{array}$} \\
\hline & \multirow{2}{*}{$\begin{array}{l}\text { No } \\
\text { OR }\end{array}$} & \multicolumn{4}{|c|}{ Yes } & \multirow{2}{*}{$\begin{array}{l}\text { No } \\
\text { OR }\end{array}$} & \multicolumn{4}{|c|}{ Yes } \\
\hline & & Estimate & SE & OR (95\% CI) & $P$ & & Estimate & SE & OR $(95 \% \mathrm{CI})$ & $P$ \\
\hline $\begin{array}{c}\text { Age, gender } \\
\text { adjusted model }\end{array}$ & $\begin{array}{c}1.00 \\
\text { [Ref] }\end{array}$ & -0.07 & 0.31 & $0.14(0.04-0.50)$ & 0.002 & $\begin{array}{l}1.00 \\
{[\text { Ref] }}\end{array}$ & -1.86 & 0.66 & $0.16(0.04-0.57)$ & 0.005 \\
\hline $\begin{array}{l}\text { Multivariable } \\
\text { adjusted model }^{\mathrm{b}}\end{array}$ & $\begin{array}{l}1.00 \\
\text { [Ref] }\end{array}$ & -2.08 & 0.77 & $0.13(0.03-0.57)$ & 0.007 & $\begin{array}{c}1.00 \\
{[\mathrm{Ref}]}\end{array}$ & -1.97 & 0.77 & $0.14(0.03-0.63)$ & 0.01 \\
\hline
\end{tabular}

OR: odds ratio; CI: confidence interval; Ref: reference.

${ }^{a}$ Two groups were classified according to the median of the total minutes per week of moderate and vigorous/strenuous activity (745 metabolic equivalents).

${ }^{b}$ Multivariable adjusted model: Adjusted for age, gender, smoking, alcohol drinking, BMI, education, and total portions of fruit and vegetable intake, census tracts and blocks, and discrepancies in survey versions for physical activity data collection. 


\section{Discussion}

In a Mexican-American cohort, subjects with total minutes per week of moderate and vigorous/strenuous activity greater than 745 METs reduced cancer risk by $87 \%$, and subjects who met physical activity guidelines of 150 moderate and vigorous minutes per week $(\geq 600$ METs) reduced cancer risk by $86 \%$ compared with their corresponding counterparts, after adjusting for age, gender, BMI, smoking and alcohol drinking status, education and total portions of fruit and vegetable intake. These data strongly indicate the potential for physical activity to have a profound effect on reported cancer risk, and they are all the more striking because there appears to be a dose effect with exceeding the guidelines being associated with even lower cancer risk.

Though only a few previous case-control studies examined the association between physical activity and cancer risk among Mexican-Americans, not all studies showed statistically significant associations. One casecontrol study [5] found that high levels of total nonoccupational activity (i.e., exercise, housework, and heavy outside work) around the time of diagnosis were associated with a greatly reduced breast cancer risk (71\%) among 720 Hispanic premenopausal women (multivariable-adjusted OR $=0.29$, 95\% CI 0.12-0.72), but not among 836 nonHispanic whites $(\mathrm{OR}=1.13,0.49-2.61)[5]$. In contrast, another case-control study reported a non-significant association (OR=0.52, 0.23-1.16) between physical activity and breast cancer risk in 798 Hispanic female cases and 924 Hispanic female controls after adjusting for other lifestyle-related factors [6]. A case-control study found that 85 Mexican female cases with 38 or more METs hours per week reduced their risk of endometrial cancer by $53 \%$ compared with 668 Mexican female controls (multivariable-adjusted OR $=0.47$, 95\% CI $=0.3-$ 0.9) in Mexico city [7], but no similar published studies were available among American Hispanic women. Although the risk reduction in our study is large, it is comparable to those (53\%-71\%) from published studies $[5,7]$. And the reference group is participants who did not meet US physical activity guidelines but no other influencing factors, therefore, our results are reasonable. Furthermore, we found that individuals with lower physical activity levels were more likely to report cancer compared with their counterparts, consistent with other findings among Mexican Americans [5,7].

Exercise appears to have a beneficial effect in reducing reported cancers in our randomly selected population based cohort [23]. To date however, the mechanism(s) remains unknown. Potential mechanisms influenced by exercise include alterations in steroid hormones [24] or insulin/insulin-like growth factors [25,26], immune modulation [27], alterations in free radical generation $[25,28]$, changes in body composition or weight, and direct effects on the tumor [23], genetic determinants (seldom monogenic, frequently polygenic) [29-31], and physical activity may contribute substantially to the healthy cell hypothesis in that healthier cells have highly effective DNA repair machinery [32,33]. Consistently, we found that subjects with lower physical activity levels had higher levels of insulin (12.59 vs $11.79 \mathrm{mg} / \mathrm{dL} ; P=0.34)$, hsCRP (4.84 vs $3.38 \mathrm{mg} / \mathrm{dL} ; P=0.01)$, HOMA-IR (3.35 vs $3.03 \mathrm{mg} / \mathrm{dL} ; P=0.16$ ), BMI (31.96 vs. $31.14 \mathrm{~kg} / \mathrm{m}^{2} ; P$
$=0.25)$, WHR (0.948 vs. $0.924 ; P=0.003)$ and body fat percentage (39.06\% vs. $35.22 \%$; $P=0.01$ ) compared to subjects with higher physical activity levels. More experimental research in both animal models and in human studies is needed to understand the basic biological mechanisms underlying the beneficial effect of physical activity on reducing the risk of cancer.

There are some methodological limitations in our research. The study was cross-sectional in design; thus, only association but not causal relationship may be inferred and it is difficult to determine whether cancer followed physical activity in time or physical activity level resulted from the outcome. Physical activity level is only reflecting the subjects' exercise status when we collecting data, thus we could not know if the cancer patients increased their physical activity level after their health providers' advice or if they decreased their physical activity level due to cancer. Prospective studies are needed to further investigate the mechanisms and even doseresponse of physical activity on cancer risk. Our longitudinal data currently being collected may contribute to that effort. The cancer identification is self-reported, and thus the biological diagnosis of cancer cannot be confirmed; however, the self-reported approach we used has been validated in one study by correlating up to $84 \%$ of self-reported cancers with pathology from medical records [34] and cancer prevalence from our results were consistent with those from our previous reports in the same cohort [35]. The sample size for cancer by specific sites were small, thus we could not explore the association between physical activity and risk for site-specific cancer; however, the expansion of this cohort provides our opportunity to examine those associations in the future. There were 99 cases of cancer included in this analysis, which is small. Our report of the large association between physical activity and cancer risk reduction are large with wide, but significant confidence intervals that are consistent with many similar studies. Given the relative low frequency of cancer most studies of the type we report are by nature smaller and therefore have wide confidence intervals, however these studies consistently demonstrate a significant effect despite relatively small sample sizes.

Our study was on a randomly selected population so there is no reason that any bias that would affect both cancer reporting and physical activity reporting to the extent that we see in our study. Thus within the statistical limits of the study ours is quite valid and should join other reports in encouraging people to increase their levels of physical activity. This study provides an initial positive effect of physical activity on cancer in Mexican Americans, and our prospective study will recruit more subjects to increase the sample size and provide a more robust estimate of the effect of physical activity. We could not completely rule out the possibility of residual confounding due to unmeasured or inadequately measured covariates, but we controlled for a large number of known covariates.

This study had several strengths. First, this is a general population-based randomly selected Mexican-American cohort, thus avoiding bias inherent in studies drawing from clinic populations or other non-randomly selected populations with established disease or mixed ethnicity. Second, detailed assessment of physical activity and information on a wide range of factors related to cancer 
was available, allowing us to get a relatively comprehensive analysis of the relevant factors. If having cancer might have increased physical activity as a result we did not observe this in our robust baseline cohort population.

In conclusion, increased moderate and vigorous physical activity significantly reduced risk of self-reported cancer after excluding the effect of other confounding factors. Therefore, physical activity might be a modifiable protective factor for which Mexican-Americans can make changes to reduce their cancer risk. Efforts need to be focused on improving physical activity intervention.

\section{Acknowledgement}

\section{Authors' contributions to manuscript}

The authors' responsibilities were as follows----JBM and SFH designed research; JBM, SPF and BR conducted research; SW analyzed data; SW, JBM, SFH and BR wrote the paper. All authors read and approved the final manuscript. None of the authors had any potential conflicts of interest to disclose.

We thank our cohort team, particularly, Rocio Uribe (Brownsville), Becky Erazo (Laredo), Ariana Garza (Harlingen) and their teams, who recruited and documented the participants. We also thank Marcela Morris and other laboratory staff for their contributions, and Christina Villarreal for administrative support. We thank Valley Baptist Medical Center, Brownsville, Texas for providing us space for our Center for Clinical and Translational Science Clinical Research Unit. We also thank the community of Brownsville, Laredo and Harlingen and the participants who so willingly participated in this study in their city.

Sources of support: This work was supported by MD000170 P20 funded from the National Center on Minority Health and Health Disparities, the Centers for Translational Science Award 1U54RR023417-01 from the National Center and the Centers for Disease Control Award RO1 DP000210-01 for Research Resources.

\section{Competing Interests}

The authors have no competing interests.

\section{Abbreviations}

BMI: Body mass index

BP: blood pressures

CCHC: Cameron County Hispanic Cohort

CI: confidence interval

CRP: C-reactive protein

DBP: diastolic blood pressures

Hb: hemoglobin

HC: hip circumference

HDLC: high density lipid cholesterol

HOMA-IR: Homeostasis model assessment insulin resistance index

IPAQ: International Physical Activity Questionnaire short-form

LDL: low-density lipoprotein
MET: metabolic equivalent

OR: odds ratio

$\mathrm{RR}$ : relative risk

SBP: systolic blood pressures

WC: waist circumference

WHR: waist-to-hip ratio

\section{References}

[1] Ennis SR, Rios-Vargas M, Albert NG. The Hispanic Population: 2010. 2011. 2010 Census Briefs.

[2] US Census Bureau. Current Population Survey, Annual Social and Economic Supplement, 2010. 2011.

[3] American Cancer Society. Cancer Facts \& Figures for Hispanics/Latinos 2012-2014 . 2012. Atlanta, American Cancer Society.

[4] Lemanne D, Cassileth B, Gubili J, "The role of physical activity in cancer prevention, treatment, recovery, and survivorship", Oncology (Williston Park), 27(6),580-585, 2013.

[5] Gilliland FD, Li YF, Baumgartner K, Crumley D, Samet JM, "Physical activity and breast cancer risk in hispanic and nonhispanic white women", Am J Epidemiol, 154(5),442-450, 2001.

[6] Slattery ML, Edwards S, Murtaugh MA, Sweeney C, Herrick J, Byers $\mathrm{T}$ et al., "Physical activity and breast cancer risk among women in the southwestern United States", Ann Epidemiol, 17(5), 342-353, 2007.

[7] Salazar-Martinez E, Lazcano-Ponce EC, Lira-Lira GG, EscuderoDe los RP, Salmeron-Castro J, Larrea F et al., "Case-control study of diabetes, obesity, physical activity and risk of endometrial cancer among Mexican women", Cancer Causes Control, 11(8), 707-711, 2000.

[8] Fisher-Hoch SP, Rentfro AR, Salinas JJ, Perez A, Brown HS, Reininger BM et al., "Socioeconomic status and prevalence of obesity and diabetes in a Mexican American community, Cameron County, Texas, 2004-2007", Prev Chronic Dis, 7(3),A53, 2010.

[9] Fisher-Hoch SP, Vatcheva KP, Laing ST, Hossain MM, Rahbar $\mathrm{MH}$, Hanis CL et al., "Missed opportunities for diagnosis and treatment of diabetes, hypertension, and hypercholesterolemia in a Mexican American population, Cameron County Hispanic Cohort, 2003-2008", Prev Chronic Dis, 9,110298, 2012.

[10] Craig CL, Marshall AL, Sjostrom M, Bauman AE, Booth ML, Ainsworth BE et al., "International physical activity questionnaire: 12-country reliability and validity", Med Sci Sports Exerc, 35(8), 1381-1395, 2003.

[11] Gofin G, Shephard RJ, "Godin leisure-time exercise questionnaire", Med Sci Sports Exerc, 29(6s),S36-S38, 2015.

[12] Reininger BM, Mitchell-Bennett L, Lee M, Gowen RZ, Barroso CS, Gay JL et al., "Tu Salud, inverted exclamation markSi Cuenta!: Exposure to a community-wide campaign and its associations with physical activity and fruit and vegetable consumption among individuals of Mexican descent", Soc Sci Med, 143,98-106, 2015.

[13] Bauman A, Bull F, Chey T, Craig CL, Ainsworth BE, Sallis JF et al., "The International Prevalence Study on Physical Activity: results from 20 countries", Int J Behav Nutr Phys Act, 6,21, 2009.

[14] Hallal PC, Gomez LF, Parra DC, Lobelo F, Mosquera J, Florindo AA et al., "Lessons learned after 10 years of IPAQ use in Brazil and Colombia", J Phys Act Health, 7 Suppl 2,S259-S264, 2010.

[15] Rauh MJ, Hovell MF, Hofstetter CR, Sallis JF, Gleghorn A, "Reliability and validity of self-reported physical activity in Latinos", Int J Epidemiol, 21(5),966-971, 1992.

[16] Dang MM. Evidence of Reliability and Validity of IPAQ for Mexican-american Adults (Order No. 1470212). [ Dissertations \& Theses @ University of Texas Health Science Center at Houston; 2009.

[17] USDHHS. 2008 Physical activity guidelines for Americans. 2008. USDHHS.

[18] Reininger BM, Wang J, Fisher-Hoch SP, Boutte A, Vatcheva K, McCormick JB, "Non-communicable diseases and preventive health behaviors: a comparison of Hispanics nationally and those living along the US-Mexico border", BMC Public Health, 15,564, 2015.

[19] US Department of Agriculture. Choose My Plate, Food Groups. 2013. US Department of Agriculture. [Accessed Sep. 9, 2015]. 
[20] US Department of Agriculture. Choose My Plate, Vegetables. 2013. US Department of Agriculture. [Accessed Sep. 9, 2015].

[21] Sun SS, Chumlea WC, Heymsfield SB, Lukaski HC, Schoeller D, Friedl $\mathrm{K}$ et al., "Development of bioelectrical impedance analysis prediction equations for body composition with the use of a multicomponent model for use in epidemiologic surveys", Am $J$ Clin Nutr, 77(2),331-340, 2003.

[22] Matthews DR, Hosker JP, Rudenski AS, Naylor BA, Treacher DF, Turner RC, "Homeostasis model assessment: insulin resistance and beta-cell function from fasting plasma glucose and insulin concentrations in man", Diabetologia, 28(7),412-419, 1985.

[23] Westerlind KC, "Physical activity and cancer prevention-mechanisms", Med Sci Sports Exerc, 35(11),1834-1840, 2003.

[24] Verkasalo PK, Thomas HV, Appleby PN, Davey GK, Key TJ, "Circulating levels of sex hormones and their relation to risk factors for breast cancer: a cross-sectional study in 1092 pre- and postmenopausal women (United Kingdom)", Cancer Causes Control, 12(1),47-59, 2001.

[25] McTiernan A, Ulrich C, Slate S, Potter J, "Physical activity and cancer etiology: associations and mechanisms", Cancer Causes Control, 9(5), 487-509, 1998.

[26] Thompson PD, Crouse SF, Goodpaster B, Kelley D, Moyna N, Pescatello L, "The acute versus the chronic response to exercise", Med Sci Sports Exerc, 33(6 Suppl),S438-S445, 2001.

[27] Shephard RJ, Verde TJ, Thomas SG, Shek P, "Physical activity and the immune system", Can J Sport Sci, 16(3),169-185, 1991.
[28] Ji LL, "Antioxidants and oxidative stress in exercise", Proc Soc Exp Biol Med, 222(3),283-292, 1999.

[29] Davis CD, Milner J, "Frontiers in nutrigenomics, proteomics, metabolomics and cancer prevention", Mutat Res, 551(1-2),51-64, 2004.

[30] Cai Z, Chiu JF, He QY, "Application of proteomics in the study of tumor metastasis", Genomics Proteomics Bioinformatics, 2(3),152-166, 2004.

[31] Harris H, "A long view of fashions in cancer research", Bioessays, 27(8),833-838, 2005.

[32] Stolk L, Perry JR, Chasman DI, He C, Mangino M, Sulem P et al., "Meta-analyses identify 13 loci associated with age at menopause and highlight DNA repair and immune pathways", Nat Genet, 44(3),260-268, 2012.

[33] Shen MR, Jones IM, Mohrenweiser H, "Nonconservative amino acid substitution variants exist at polymorphic frequency in DNA repair genes in healthy humans", Cancer Res, 58(4),604-608, 1998.

[34] Airewele G, Adatto P, Cunningham J, Mastromarino C, Spencer C, Sharp $\mathrm{M}$ et al., "Family history of cancer in patients with glioma: a validation study of accuracy", J Natl Cancer Inst, 90(7), 543-544, 1998.

[35] Garza A, Vatcheva P, Pan JJ, Rahbar MH, Fallon MB, McCormick JB et al., "Liver and Other Gastrointestinal Cancers Are Frequent in Mexican Americans", $J$ Racial and Ethnic Health Disparities,1-10, 2014. 